\title{
Propuesta personal de actualización para el tratamiento de la hipercolesterolemia en Colombia
}

\author{
Alonso Merchán V. ${ }^{\mathrm{a}, \mathrm{b}}$ \\ a Fundación Clínica Shaio, Bogotá, Colombia \\ b Capítulo Colombia de la Red Iberoamericana de Hipercolesterolemia Familiar, Bogotá, Colombia
}

Recibido el 8 de mayo de 2017; aceptado el 25 de agosto de 2017

Disponible en Internet el 10 de noviembre de 2017

\section{PALABRAS CLAVE \\ Inhibidores de la \\ PCK9; \\ Evolocumab; \\ Dislipidemia; \\ Hipercolesterolemia \\ familiar; \\ Metas en el cLDL}

\author{
KEYWORDS \\ PCK9 inhibitors; \\ Evolocumab; \\ Dyslipidemia; \\ Familial hypercholes- \\ terolemia; \\ LDLC goals
}

\begin{abstract}
Resumen En 2014, Colombia publicó una guía para el diagnóstico y tratamiento de las dislipidemias en adultos, la cual fue similar a la del Colegio Americano de Cardiología/Asociación Americana del Corazón ( $\mathrm{ACC} / \mathrm{AHA}$ ) en lo referente a la rigidez en la revisión sistemática de la evidencia con los inhibidores de la hidroxi-metil-glutaril coenzima A (estatinas). El resultado de la revisión fue no utilizar metas en el colesterol contenido en la lipoproteína de muy baja densidad (CLDL por su sigla en inglés), sino porcentaje en la reducción del cLDL basal, empleándose para su logro las dosis más altas de las estatinas de alta intensidad. El planteamiento creó controversia y a pesar de reconocerse la evidencia, se argumentó que el concepto de metas se podría deducir de los experimentos clínicos y facilitaría a médicos y pacientes su aplicación en la práctica clínica. En los últimos años, las guías internacionales sobre dislipidemia enfatizan en las metas en el CLDL, se han publicado nuevos experimentos clínicos y han entrado al mercado novedosos fármacos hipolipemiantes, hallazgos que justifican actualizar el tratamiento de la hipercolesterolemia en Colombia, y para ello, se hace una propuesta personal, y se reconoce que requiere ser consensada. De otra parte, los recientes hallazgos confirman aún más la importancia del colesterol como factor de riesgo para enfermedad cardiovascular y reafirman el concepto que cuanto más bajo tenga un individuo el colesterol, menor probabilidad de eventos cardiovasculares.

(c) 2017 Publicado por Elsevier España, S.L.U. en nombre de Sociedad Colombiana de Cardiología y Cirugía Cardiovascular. Este es un artículo Open Access bajo la licencia CC BY-NC-ND (http:// creativecommons.org/licenses/by-nc-nd/4.0/).
\end{abstract}

\section{Personal proposal for updating the treatment of hypercholesterolemia in Colombia}

Abstract In 2014, Colombia published guidelines for the diagnosis and treatment of dyslipidemias in adults, which were similar to those published by the American College of Cardiology/American Heart Association (ACC/AHA) with regard to their rigidity in the systematic review of the evidence in hydroxymethylglutaryl coenzyme A inhibitors (statins). The result of the review was to not set goals for the cholesterol contained in very low density lipoproteins $(L D L C)$, but rather a percentage reduction in basal LDLc, using the highest doses

Correo electrónico: alomerchan@hotmail.com 
of high-intensity statins to accomplish this. This proposal created controversy, and in spite of recognizing the evidence, it was argued that the concept of goals could be deduced from the clinical studies, and would make application to clinical practice easier for physicians and patients. In the last few years, international guidelines on dyslipidemias have emphasized LDLc goals, new clinical studies have been published, and novel hypolipidemic medications have appeared on the market. These findings warrant updating hypercholesterolemia treatment in Colombia, and a personal proposal is presented for this purpose, recognizing that a consensus must be reached. Additionally, the recent findings further confirm the importance of cholesterol as a risk factor for cardiovascular disease, and reaffirm that the lower an individual's cholesterol, the lower the probability of cardiovascular events.

(c) 2017 Published by Elsevier España, S.L.U. on behalf of Sociedad Colombiana de Cardiología y Cirugía Cardiovascular. This is an open access article under the CC BY-NC-ND license (http: / / creativecommons.org/licenses/by-nc-nd/4.0/).

\section{Introducción}

Hay consenso en considerar el cLDL como el principal objetivo terapéutico para reducir eventos cardiovasculares, y que cuanto más se reduzca, menor número de eventos se presentarán, criterio conocido como "cuanto más bajo el cLDL, mejor'”. Al respecto surgen varias preguntas:

1) ¿Cuál es la evidencia?

2) ¿Cuál es la meta en el cLDL y en qué población?

3) ¿Con cuál o cuáles fármacos se lograría?

4) ¿Cuál es la seguridad, la adherencia a las estatinas y el porcentaje de pacientes que llegan a metas?

5) ¿Cuál es la variabilidad individual de la respuesta en el cLDL con estatinas?

6) ¿Reducen los nuevos hipolipemiantes significativamente los eventos cardiovasculares (ECV) y son seguros?

7) ¿Son deletéreos los niveles muy bajos en el cLDL?

8) ¿Cuál es la importancia clínica de la regresión de la placa aterosclerótica?

9) ¿Cuánto más temprano y prolongado el tratamiento, mejor?

Estas preguntas se resolverán de manera extractada y con base en las respuestas se hará una propuesta personal para el tratamiento de la hipercolesterolemia, aplicable a Colombia, aceptando que se debe discutir.

\section{El CLDL, los eventos cardiovasculares y el concepto de "no metas" en el cLDL}

Los metanálisis que han comparado estatinas versus placebo, enseñan que reducir el cLDL en $40 \mathrm{mg} / \mathrm{dL}$, disminuye aproximadamente en un $22 \%$ la aparición de $\mathrm{ECV}^{1-3}$ y que extrapolar la reducción del cLDL en $80 \mathrm{mg} / \mathrm{dl}$, se traduce en un descenso de estos eventos en un $40 \%{ }^{1}$. El metanálisis de estudios de prevención secundaria, que comparó altas dosis de estatinas con dosis bajas, encontró que alcanzar cifras promedio de $70 \mathrm{mg} / \mathrm{dl}$ en el cLDL, reduce más ECV comparado con cifras promedio de $100 \mathrm{mg} / \mathrm{dl}^{4}$.
Los estudios correspondientes a estos metaanálisis ${ }^{1-4}$, no fueron diseñados para buscar metas sino porcentaje en la reducción del cLDL con estatinas, y es de acuerdo con esta rigurosa revisión sistemática, que nace la propuesta de reducir más del $50 \%$ del cLDL basal y no metas, y explica la posición de la guía del $A C C / \mathrm{AHA}^{5}$ y de la guía colombiana ${ }^{6}$. La Asociación Americana de Diabetes (ADA), se alinea con este concepto ${ }^{7}$ y la guía de la Sociedad de Cardiología de Canadá ${ }^{8}$, acepta reducir el CLDL basal más del $50 \%$ o metas en el CLDL.

De los metanálisis mencionados ${ }^{1-4}$, se puede deducir, aunque sin la rigurosidad de la evidencia, metas en el cLDL. Las siguientes guías aceptan este criterio.

La Asociación Nacional de Lípidos de los Estados Unidos (NLA), considera un cLDL menor de $70 \mathrm{mg} / \mathrm{dl}$ en muy alto riesgo y menor de $100 \mathrm{mg} / \mathrm{dl}$ en otros riesgos 9 .

La Sociedad Europea de Cardiología/Sociedad Europea de Aterosclerosis ${ }^{10}$, también busca una meta menor de $70 \mathrm{mg} / \mathrm{dl}$ en muy alto riesgo (enfermedad cardiovascular documentada, diabetes mellitus-DM- con compromiso de órgano blanco $u$ otro factor de riesgo asociado, y la enfermedad renal crónica -ERC- con eTFG menor de $30 \mathrm{ml} / \mathrm{min} / 1,73 \mathrm{~m}^{2}$ ). Plantea una meta menor de $100 \mathrm{mg} / \mathrm{dl}$ en riesgo alto (hipercolesterolemia familiar - HF-), factor de riesgo no controlado, DM sin las condiciones anteriores y ERC con eTFG entre $30-59 \mathrm{ml} / \mathrm{min} / 1,73 \mathrm{~m}^{2}$; y finalmente, sugiere una meta menor de $115 \mathrm{mg} / \mathrm{dl}$ en riesgo bajo a moderado (SCORE menor del 5\%).

El Colegio Americano de Cardiología (ACC), en su documento sobre las no-estatinas en pacientes con enfermedad cardiovascular aterosclerótica $(E C V A)^{11}$, propone metas menores de $100 \mathrm{mg} / \mathrm{dl}$ en las personas con ECVA no complicada y menor de $70 \mathrm{mg} / \mathrm{dl}$ cuando la ECVA se asocia a DM o HF. El ACC deja a consideración el uso de los anticuerpos monoclonales o inhibidores de la proproteína convertasa subtilisina/kexina tipo 9 (i PCSK9), cuando no se logran las metas mencionadas y considera como segunda línea a los secuestrantes de ácidos biliares $(\mathrm{SAB})^{11}$.

La Asociación Americana de Endocrinólogos Clínicos/Colegio Americano de Endocrinólogos (AACE/ACE) ${ }^{12}$, adiciona el "riesgo extremo" (enfermedad aterosclerótica cardiovascular progresiva, enfermedad cardiovascular más 
DM o ERC estados 3 y 4 y a las personas con HF heterocigota -HFHe- más historia de enfermedad coronaria prematura) y formulan para este grupo un cLDL menor de $55 \mathrm{mg} / \mathrm{dl}$. Para riesgos muy altos y altos, el objetivo son cifras menores de 70 y $100 \mathrm{mg} / \mathrm{dl}$, respectivamente.

A la fecha, se acepta más el criterio de metas en el cLDL por la facilidad y entendimiento entre médicos y pacientes respecto al tratamiento y para aprovechar que este concepto se conocía desde el ATP III ${ }^{13}$.

\section{Seguridad, adherencia a las estatinas y logro de metas}

A pesar de la eficacia de las estatinas en la reducción de eventos cardiovasculares y su relevante relación beneficioriesgo, se les ha imputado una baja seguridad errónea. La evidencia sobre este tópico demuestra que son fármacos con pocos efectos colaterales, si se utilizan de manera adecuada ${ }^{14}$. Infortunadamente, su seguridad es mal interpretada por médicos y pacientes y agravada por la desinformación del periodismo, lo cual explica la adherencia reducida ${ }^{15}$. El evento adverso más sobre-diagnosticado de manera errónea son los síntomas musculares asociados a las estatinas (su prevalencia real está cerca al 5\%) ${ }^{16}$. El logro de meta en el cLDL menor de $70 \mathrm{mg} / \mathrm{dl}$ en personas de alto riesgo, suele estar entre el $20 \%$ y $40 \%{ }^{14,15}$. Un estudio llevado a cabo en una institución médica de Bogotá, encontró en pacientes con enfermedad coronaria, que un $22 \%$ tenían un CLDL menor de $70 \mathrm{mg} / \mathrm{dl}^{17}$.

\section{La variabilidad individual de la respuesta sobre el cLDL con estatinas}

La alta variabilidad de las estatinas sobre el CLDL se observa en el subanálisis del estudio Júpiter ${ }^{18}$, el cual es un experimento en personas de prevención primaria, que recibieron rosuvastatina $20 \mathrm{mg} /$ día versus placebo; su subanálisis ${ }^{19}$ mostró que en $46 \%$ de los pacientes se redujo el cLDL en más del $50 \%$, $43 \%$ entre el 0 y el $50 \%$ y en $11 \%$ no cambió o aumentó. Lo anterior muestra la respuesta interindividual e inesperada del cLDL con el uso de estatinas y resalta la importancia de medir el cLDL a los dos o tres meses de iniciarlas, con el objetivo de ajustar las metas.

\section{El cLDL y los eventos cardiovasculares con hipolipemiantes no-estatinas}

Se aceptan como hipolipemiantes no-estatinas a los secuestrantes de ácidos biliares (SAB), el ácido nicotínico-niacina (AN), los fibratos, los ácidos grasos omega-3 (AGO-3), el ezetimibe, los i PCSK9, el lomitapide y el mipomersen; estos dos últimos se utilizan en hipercolesterolemia familiar homocigota y no se analizarán en esta revisión.

Cuando a pesar de dosis altas de estatinas de alta efectividad (atorvastatina, rosuvastatina), no se llega a metas en el CLDL (de acuerdo con el riesgo de ECV de un individuo), se requiere asociar a la estatina un hipolipemiante no-estatina

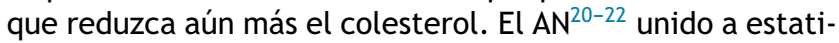
nas reduce el CLDL en $19 \% 22$ mientras que los $S A B$ lo reducen aproximadamente en $10-15 \%$. Los fibratos ${ }^{20,23}$ y los AGO- $3^{20}$, asociados a estatinas, tienen repercusión nula sobre el cLDL. Estos cuatro hipolipemiantes tienen evidencia reducida y controversial sobre la disminución de ECV, en parte por el momento histórico en que se realizaron los estudios, tiempo en el que no se utilizaban rutinariamente las estatinas ni otros fármacos cardiovasculares ${ }^{10-12,20}$; son interesantes los resultados de un metanálisis con $\mathrm{AN}$ en el cual se demuestra reducción significativa de ECV en monoterapia o en conjunto con estatinas ${ }^{29}$. La tolerancia y adherencia del AN y los SAB es muy baja, debido a sus efectos gastrointestinales, especialmente con la colestiramina, que es la resina disponible en Colombia ${ }^{10-12,20}$ y por los eventos adversos sobre piel y sistema digestivo con el $\mathrm{AN}^{20-22}$. El colesevelan, disponible en otros países, es mejor tolerado y reduce el CLDL $15-20 \%$, pero no tiene estudios que demuestren reducción de ECV; se propone como tercera línea en las guías europea ${ }^{10}$, del $\mathrm{ACC}^{11}$ y de la AACE/ACE ${ }^{12}$.

Dentro de los hipolipemiantes no-estatinas, los que tienen alto impacto sobre el cLDL, buena tolerancia y adherencia, bajos efectos colaterales y reducción significativa de ECV, son el ezetimibe y los i PCSK9, fármacos que se estudiarán a continuación.

\section{cLDL, eventos cardiovasculares y ezetimibe}

El ezetimibe como monoterapia disminuye el cLDL en aproximadamente $20 \%$ y asociado a estatinas logra una reducción adicional del $19-24 \%{ }^{24,25}$. El estudio IMPROVE-IT ${ }^{25}$, hecho en pacientes de prevención secundaria, demostró que la simvastatina asociada a ezetimibe alcanzó un cLDL de $53 \mathrm{mg} / \mathrm{dl}$ mientras que la simvastatina como monoterapia, logró reducirlo en $69 \mathrm{mg} / \mathrm{dl}$; esta diferencia, dio como resultado una reducción pequeña pero significativa en ECV del $7 \%$. El hallazgo apoya el criterio de cifras de CLDL menores de $70 \mathrm{mg} / \mathrm{dl}$ para reducir dichos eventos en personas de alto riesgo (cercano a $50 \mathrm{mg} / \mathrm{dl}$ ) y posiciona al ezetimibe como el primer hipolipemiante no-estatina en demostrar tal beneficio. Combinado con altas dosis de las estatinas más efectivas, es una alternativa para el logro de una mayor reducción en el cLDL o en pacientes que no toleraran altas dosis de estatinas.

\section{El cLDL, los eventos cardiovasculares y los inhibidores de la PCSK9}

En los últimos años, dentro del armamentario de los hipolipemiantes aparecen los i PCSK9, moléculas que corresponden al grupo de los anticuerpos monoclonales (ACsM). De forma resumida, a nivel hepático, la PCSK9 se adhiere a los receptores de la LDL (RLDL) y los lleva a su degradación. Los i PCSK9 se unen a la PCSK9 sérica y evitan la degradación en mención, lográndose más RLDL disponibles, que captarán más CLDL y así disminuirán los niveles plasmáticos de CLDL $^{26-28}$. Los dos ACsM aprobados por las agencias internacionales de salud (FDA y EMA, entre otras, y colombiana -INVIMA), son el evolocumab y el alirocumab, ambos para uso subcutáneo quincenal (dosis aprobada en Colombia) o mensual.

El estudio FOURIER (Further cardiovascular OUtcomes Research with PCSK9 Inhibition in subjects with Elevated Risk), es el primero en dar a conocer la eficacia del 
evolocumab en puntos duros ${ }^{30}$. Ingresaron al estudio 27.500 personas con antecedente de infarto miocárdico, ataque cerebrovascular isquémico (ACV) y enfermedad arterial periférica, quienes a pesar del tratamiento con estatinas/ezetimibe persistían con cLDL mayor de $70 \mathrm{mg} / \mathrm{dl}$. Se analizó la respuesta cardiovascular de evolocumab versus placebo de evolocumab, y la continuación con el tratamiento hipolipemiante inicial para ambos brazos. El grupo con evolocumab logró una reducción del cLDL respecto al basal del 59\% (de una mediana de $92 \mathrm{mg} / \mathrm{dl}$ a una mediana de $30 \mathrm{mg} / \mathrm{dl}$ ). La reducción del riesgo relativo (RRR) del punto final primario (muerte cardiovascular, infarto de miocardio, ACV, hospitalización por angina inestable y revascularización miocárdica) fue significativa en $15 \%$, con una significativa RRR de infarto de miocardio en $27 \%$, ACV en $25 \%$ y RVM en $22 \%$. Es importante anotar que la población del FOURIER tenía un alto porcentaje de personas con hipertensión, diabetes y tabaquismo. Se esperan los desenlaces del estudio ODYSSEY OUTCOMES, que analizará al alirocumab en 18.000 pacientes, también de alto riesgo (posterior a síndrome coronario agudo) ${ }^{31}$.

La seguridad en relación con eventos adversos serios, es para evolocumab a 2.2 años con los estudios FOURIER ${ }^{30}$, OSLER-1 a 4 años de seguimiento ${ }^{32}$ y EBBINGHAUS a 2.2 años el cual no reportó compromiso neurocognitivo ${ }^{33}$; los estudios de seguridad con alirocumab son similares ${ }^{34}$. De otra parte, los valores muy bajos en el cLDL no se asocian con eventos adversos como se observa con evolocumab ${ }^{30}$ y alirocumab ${ }^{35}$. De manera similar, este último reduce el $\mathrm{cLDL}$ en $60 \%{ }^{34}$.

A partir de los estudios realizados con evolocumab y alirocumab se puede deducir que:

1. Son una opción para pacientes de alto riesgo que no llegan a metas en el cLDL a pesar de dosis altas de estatinas de alta intensidad, asociadas a ezetimibe, estrictos cambios en el estilo de vida (CEV) y adherencia estricta con lo mencionado.

2. En muy alto riesgo, la reducción de ECV se logra con niveles bastante inferiores a los $70 \mathrm{mg} / \mathrm{dl}$ que habitualmente se recomiendan.

3. La seguridad con los i PCSK9, al menos a 4 años, es alta.

4. Los niveles muy bajos de cLDL igualmente son seguros para el paciente.

\section{Importancia clínica de la regresión de la placa aterosclerótica}

Los estudios de regresión de la placa aterosclerótica, mediante ultrasonido intravascular (IVUS) ${ }^{36-39}$, demuestran que la regresión se logra con el uso de estatinas ${ }^{36-38}$ o estatina asociada a ezetimibe ${ }^{39}$, toda vez que el cLDL esté por debajo de $60 \mathrm{mg} / \mathrm{dl}$; respuesta similar arroja el estudio GLAGOV con evolocumab ${ }^{40}$. De otra parte, la regresión de la placa impacta sobre los ECV, de acuerdo con un subanálisis del estudio SATURN ${ }^{41}$, hallazgos que apoyan el concepto de metas en el cLDL menores de $70 \mathrm{mg} / \mathrm{dl}$.

\section{Posición de las guías con la incorporación de los i PCSK9}

Las guías recientemente publicadas sobre dislipidemia consideran a los i PCSK9 como una alternativa a considerar en determinada población ${ }^{8-11}$. Específicamente, se han publicado cuatro documentos que analizan y consideran el uso de los i PCSK9 ${ }^{11,42-44}$.

Respecto al uso de los i PCSK9, el $\mathrm{ACC}^{11}$ propone dos grupos: 1) EACV con comorbilidades [DM, síndrome coronario agudo (SCA) menor de tres meses, HF, evento coronario recidivante mientras se toma una estatina, factor de riesgo mal controlado, Lp(a) mayor de $50 \mathrm{mg} / \mathrm{dl}$ y ERC] y 2) EACV sin las comorbilidades mencionadas.

Para el primer grupo proponen una meta en el cDL menor de $70 \mathrm{mg} / \mathrm{dl}$ y en el segundo grupo menor de $100 \mathrm{mg} / \mathrm{dl}$. Si no se logran estas metas con altas dosis de estatinas de alta intensidad, ezetimibe, CEV, SAB y supervisión de la adherencia, consideran la asociación de los i PCSK9.

La Sociedad Europea de Cardiología/Sociedad Europea de Aterosclerosis, presentan cuatro grupos ${ }^{42}$ : 1) muy alto riesgo (EACV, DM con daño de órgano blanco u otro factor de riesgo asociado, rápida progresión de la EACV), 2) HFHe, 3) Hipercolesterolemia familiar homocigota (HFHo) y 4) Intolerantes a las estatinas que correspondan a los tres grupos antes mencionados. En los casos en que el cLDL persista mayor de $140 \mathrm{mg} / \mathrm{dl}$ a pesar de las medidas mencionadas (exceptuando SAB), o de $100 \mathrm{mg} / \mathrm{dl}$ para los casos de EACV de rápida progresión, consideran iniciar i PCSK9. En HF si no llegan a metas menores de $175 \mathrm{mg} / \mathrm{dl}$, proponen los $\mathrm{i}$ PCSK9 ${ }^{42}$.

La Sociedad Canadiense de Cardiología ${ }^{43}$, revisa los estudios con i PCSK9 y plantea sus indicaciones, que son similares a las otras propuestas.

Las recomendaciones más recientes sobre el uso de los i PCSK9, corresponden a la Asociación Nacional de Lípidos (NLA) de Estados Unidos ${ }^{44}$, que tiene la particularidad de considerar los resultados del estudio FOURIER ${ }^{30}$. La NLA contempla tres grupos para utilizar i PCSK9: 1) EACV, 2) HF, 3 ) intolerantes a las estatinas, y propone metas en el cLDL de manera similar a las otras propuestas, con asociación de i PCSK9 si no se llega a las metas.

\section{Cuanto más temprano y prolongado el tratamiento, mejor}

A excepción de los pacientes con $\mathrm{HF}^{45}$, reducir eventos cardiovasculares con la administración de hipolipemiantes desde temprana edad, no es claro; sin embargo, un cLDL bajo de forma natural, por polimorfismo o mutaciones, impacta en la disminución de ECV, comparado con el grupo control sin mutaciones y con cLDL más altos ${ }^{46}$. Se acepta que los bajos niveles plasmáticos de cLDL desde temprana edad, disminuyen la carga aterogénica durante toda la vida ${ }^{46}$. De otra parte, la reducción de eventos será mayor con el transcurso del tiempo, por su efecto acumulativo (no solo a 5 años, sino a 40 años) y dependerá del riesgo del individuo al inicio de la terapia, al nivel basal del cLDL al iniciar el hipolipemiante, la magnitud en la reducción del CLDL y la duración sin interrupción del tratamiento ${ }^{47}$. 


\section{Propuesta para metas en el cLDL según el riesgo cardiovascular}

Parcialmente, con base en la guía colombiana sobre dislipidemia 6 , la revisión colombiana sobre $\mathrm{HF}^{45}$, el resultado del IMPROVE-IT ${ }^{21}$, estudios de regresión de placa ${ }^{36-40}$, resultados del experimento clínico con i PCSK9 ${ }^{30}$, las propuestas que consideran metas bajas en el $\mathrm{CLDL}^{9-12}$, las recomendaciones que hacen las diferentes sociedades científicas sobre el uso de los i PCSK9 ${ }^{11,42-44}$ y la deducción de metas en HF basadas en el registro SAFEHEART ${ }^{48}$, se hace una propuesta para las metas en el CLDL de acuerdo con el riesgo cardiovascular, la cual requiere ser sometida a consenso (tabla 1).

Tabla 1 Categorías de riesgo cardiovascular y metas en el $\mathrm{cLDL}(\mathrm{mg} / \mathrm{dl})$

\begin{tabular}{l}
\hline Categoría de riesgo cardiovascular \\
$\begin{array}{l}\text { CLDL } \\
\text { Riesgo muy alto ("'riesgo extremo') }\end{array}$ \\
- EACV (IM, SCA, RVM, EAP, EAC, AA, \\
ACV isquémico) asociada a \\
comorbilidades: DM, HF, evento \\
coronario recidivante a pesar \\
de altas dosis de estatinas \\
efectivas y cLDL<70 mg/dl \\
Riesgo alto \\
- EACV no complicada, es decir, sin \\
las comorbilidades anteriores \\
- HFHe asociada a DM o a varios \\
factores de riesgo (HTA, obesidad, \\
tabaquismo) 45,48 \\
- HFHo 45 \\
- DM en mayores de 40 años más otro \\
factor de riesgo y cLDL \\
> de \\
70 mg/dl ${ }^{6}$
\end{tabular}

Tabla 1 (continuación)

\begin{tabular}{ll}
\hline Categoría de riesgo cardiovascular & $\begin{array}{c}\text { Meta } \\
\text { CLDL }\end{array}$ \\
\hline - DM en menores de 40 años, & \\
sin otro factor de riesgo o daño \\
de
\end{tabular}

EACV: enfermedad aterosclerótica cardiovascular. IM: infarto de miocardio. SCA: síndrome coronario agudo. RVM: revascularización miocárdica. EAP: enfermedad arterial periférica. EAC: enfermedad arterial carotídea. AA: aneurisma aórtico. ACV: ataque cerebrovascular. DM: diabetes mellitus, HF: hipercolesterolemia familiar. HFHe: hipercolesterolemia familiar heterocigota. HFHo: hipercolesterolemia familiar homocigota. ERC: enfermedad renal crónica. CEV: cambios en el estilo de vida.

\section{Tratamiento para el logro de metas en el cLDL}

Para la consecución de las metas mencionadas en la tabla 1, inicialmente se deben reunir las siguientes condiciones:

Tabla 2 Indicaciones actuales de los i PCSK9 para lograr metas en CLDL, según el riesgo cardiovascular

\begin{tabular}{|c|c|c|}
\hline $\begin{array}{l}\text { Grupo de riesgo } \\
\text { cardiovascular } \\
\text { (tabla 1) }\end{array}$ & $\begin{array}{l}\text { Meta } \\
\text { recomendada } \\
\text { en el cLDL }\end{array}$ & $\begin{array}{l}\text { Recomendado } \\
\text { el i PCSK9 }\end{array}$ \\
\hline $\begin{array}{l}\text { Riesgo muy alto } \\
\text { (riesgo extremo) }\end{array}$ & $\begin{array}{l}\text { Menor de } \\
50 \mathrm{mg} / \mathrm{dl}^{*}\end{array}$ & Sí \\
\hline \multicolumn{3}{|l|}{ Riesgo alto } \\
\hline EACV no & Menor de & Sí \\
\hline complicada & $70 \mathrm{mg} / \mathrm{dl}^{*}$ & \\
\hline HFHe asociada a & Menor de & Sí \\
\hline \multicolumn{3}{|l|}{$\begin{array}{l}\text { factores } \\
\text { de riesgo (HTA, } \\
\text { tabaquismo, } \\
\text { obesos) }\end{array}$} \\
\hline HFHo & $\begin{array}{l}\text { Menor de } \\
70 \mathrm{mg} / \mathrm{dl}^{*}\end{array}$ & Evolocumab \\
\hline \multicolumn{3}{|l|}{ Riesgo moderado } \\
\hline $\begin{array}{l}\text { HFHe sin EACV, } \\
\text { ni DM ni otro } \\
\text { factor } \\
\text { de riesgo }\end{array}$ & $\begin{array}{l}\text { Menor de } \\
100 \mathrm{mg} / \mathrm{dl}^{*}\end{array}$ & Sí \\
\hline \multicolumn{3}{|c|}{$\begin{array}{l}\text { "Se deben reunir las cuatro condiciones mencionadas en el } \\
\text { texto. } \\
{ }^{* *} \text { Excepto en los casos con RLDL ausente o nulo. } \\
\text { *** Podría considerarse el lomitapide en casos específicos. Remi- } \\
\text { tir a pediatra experto en HF. } \\
\text { tinas (síntomas musculares asociados a las estatinas) siempre } \\
\text { y cuando correspondan a los casos de indicaciones para los } \\
\text { PCSK9, considerados en esta tabla. }\end{array}$} \\
\hline
\end{tabular}


a) Utilizar las dosis más altas de las estatinas más efectivas (atorvastatina o rosuvastatina).

b) Si no se llega a metas, asociar ezetimibe $10 \mathrm{mg} /$ día (existen asociaciones fijas de estos dos hipolipemiantes).

c) Hacer cambios estrictos en cuanto a estilo de vida.

d) Asegurarse de que haya adherencia a las anteriores recomendaciones.

Si con lo anterior no se logran las metas, se considera la prescripción de los i PCSK9, previa verificación de la existencia de evidencia clínica para usarlos, el grupo de riesgo cardiovascular al que pertenecen los pacientes, el criterio médico, las condiciones clínicas, la expectativa de vida del individuo, el costo-beneficio de la terapia y las preferencias del paciente (tabla 2).

\section{Conclusiones}

Los resultados favorables de los experimentos clínicos recientes, la evidencia en la reducción de ECV con i PCSK9, lo estricto respecto a metas bajas en el cLDL y las últimas recomendaciones para la prescripción de i PCSK9, hacen razonable que se considere actualizar el tratamiento de la hipercolesterolemia para Colombia. A la fecha, las metas en el CLDL en personas de alto riesgo son más bajas que lo propuesto en años anteriores y para su logro, además de las altas dosis de estatinas de alta efectividad, el ezetimibe y los CEV, aparece la opción de los ACsM (i PCSK9, hipolipemiante que se utiliza en una población determinada). La evidencia es suficiente para enfatizar en el axioma, "cuanto más bajo el cLDL mejor", sin olvidar el otro axioma, y "cuanto más temprano y más prolongado el tratamiento, mejor'. De otra parte, los estudios recientes mencionados confirman aún más la importancia del colesterol como factor de riesgo para enfermedad cardiovascular y reafirman el concepto que cuanto más bajo tenga un individuo el colesterol, menor probabilidad de eventos cardiovasculares.

\section{Conflicto de intereses}

Los autores declaran no tener ningún conflicto de intereses.

\section{Bibliografía}

1. Cholesterol Treatment Trialist's (CTT) Collaboration. Efficacy and safety of more intensive lowering of LDL colesterol: a metaanalysis of data from 170000 participants in 26 randomized trials. Lancet. 2010;376:1670-81.

2. Cholesterol Treatment Trialists (CTT). The effects of lowering LDL colesterol with statins therapy in people at low risk of vascular disease: meta-analysis of individual data from 27 randomised trials. Lancet. 2012;380:581-90.

3. Cholesterol Treatment Trialists' (CTT) Collaboration. Efficacy and safety of LDL-lowering therapy among men and women: meta-analysis of individual data from 174000 participants in 27 randomised trials. Lancet. 2015;385:1397-405.

4. Cannon CP, Steinberg B, Murphy S, et al. Meta-analysis of cardiovascular outcomes trials comparing intensive versus moderate statin therapy. J Am Coll Cardiol. 2006;48:438-45.

5. Stone NJ, Robinson JG, Lichtenstein AH, et al. 2013 ACC/AHA guideline on the treatment of blood cholesterol to reduce atherosclerosis cardiovascular risk in adults: a report of the American College of Cardiology/American Heart Association task force on practice guidelines. J Am Coll Cardiol. 2014;63:2889-934.

6. Muñoz O, García A, Fernández D, et al. Guía de práctica clínica para la prevención, detección temprana, diagnóstico, tratamiento y seguimiento de las dislipidemias en la población mayor de 18 años. Acta Med Colomb. 2014;39 supl. 2:5-27.

7. American Diabetes Association (ADA). Standars of medical care in diabetes-2017-. Diabetes Care. 2017;40 suppl 1:S79-81.

8. Anderson JJ, Grégoire J, Pearson GJ, et al. 2016 Canadian Cardiovascular Society guidelines for the management of dyslipidemia for the prevention of cardiovascular disease in the adult. Can J Cardiol. 2016;32:1263-82.

9. Jacobson T, Ito MK, Pharma D, et al. National Lipid Association recommendations for patient-centered management of dyslipidemia: part 1-full report. J Clin Lipidol. 2015;9:129-69.

10. Catapano AL, Grahan I, DeBacker G, et al. 2016 ESC/EAS guidelines for the management of dyslipidemias. Eur Heart J. 2016;37:2999-3058.

11. Lloyd-Jones DM, Morris PB, Birtcher KK, et al. 2016 ACC expert consensus decisión pathway on the role of non-statins therapies for LDL-cholesterol lowering in the management of atherosclerotic cardiovascular disease risk. J Am Coll Cardiol. 2016;68:92-125.

12. Garber AJ, Abrahamson MJ, Barzilay JI, et al. Consensus statement by the American Association of Clinical Endocrinologists and American College of Endocrinology (AAEC/ACE) on the comprehensivety type 2 diabetes management algorithm-2017 executive summary. Endocrin Pract. 2017;23:207-38.

13. Expert panel on detection, evaluation, and treatment of high blood cholesterol in adults. Executive summary of the third report of the national Cholesterol Education Program (NCEP) expert panel on detection, evaluation, and treatment of high blood colesterol in adultas (Adult Treatment Panel III). JAMA. 2001;285:2486-97.

14. Collins R, Reith Ch, Emberson J, et al. Interpretation of the evidence for the efficacy and safety of statins therapy. Lancet. 2016;388:2532-61.

15. Heidenreich PA, Albert NM, Chan PS, et al. 2015 ACC/AHA focused Update of secondary prevention lipid perfomance measure. A report of the ACC/AHA task forcé. J Am Coll Cardiol. 2016;67:558-87.

16. Stroes ES, Thompson PD, Corsini A, et al. European Atherosclerosis Society Consensus Panel Statin-associated muscle symptoms: impact on statin therapy-European Atherosclerosis Society Consensus Panel Statement on Assessment, Aetiology and Management. Eur Heart J. 2015;36:1012-22.

17. Merchan A, Jaramillo C, Mendoza F, et al. ¿Se están alcanzando las metas en el perfil lipídico después de enfermedad coronaria? Rev Colomb Cardiol. 2008;18:262-7.

18. Ridker PM, Danielson E, Fonseca FAH, et al. Rosuvastatin to prevent vascular events in men and women with elevated Creactive protein. New Engl J Med. 2008;359:2195-207.

19. Ridker PM, Mora S, Rose L, on behalf of the Jupiter. Percent reduction in LDL colesterol following high-intensity statin therapy: potential implications of emerging lipid-lowering agents. Eur Heart J. 2016;37:1373-9.

20. García A, Muñoz O, Fernandez D, et al. Alternativas terapéuticas al manejo farmacológico con estatinas en adultos con dislipidemia. Revisión sistemática de la literatura y recomendaciones generales. Rev Colomb Cardiol. 2015;22:179-86.

21. The AIM-HIGH Investigators. Niacin in patients with low HDL cholesterol levels receiving intensive statin therapy. New Engl J Med. 2011;365:2255-67.

22. The HPS2-THRIVE Collaborative Group. Effects of extended release niacin with laropiprant in high-risk patients. New Engl J Med. 2014;371:2255-67. 
23. The, ACCORD, Study Group. Effects of combination lipid therapy in type 2 diabetes mellitus. New Engl J Med. 2010;362: 1563-74.

24. Ballantyne C, Hori J, Notarbartolo A, et al. Effect of ezetimibe coadministred with atorvastatin in 628 patients with primary hypercholesterolemia: a prospective, randomized, double-blind trial. Circulation. 2003;107:2409-15.

25. Cannon C, Blazing M, Giuglino RP, et al., For the IMPROVEIT Investigators. Ezetimibe added to statin therapy after acute coronary síndromes. New Engl J Med. 2015;372: 2387-97.

26. Seidah N, Benjannes S, Wickham L, et al. The secretory proprotein Convertase neural apoptosis-regulated convertase 1 (NARC-1): liver regeneration and neuronal differentiation. Proc Natl Acad Sci. USA. 2003;100:928-33.

27. Abifadel M, Varret M, Rabés JP, Allard D, Duguerram K, et al. Mutations in PCSK9 cause autosomal dominant hypercholesterolemia. Nat Genet. 2003;34:154-6.

28. Cohen J, Pertsemildis A, Kotowski I, et al. Low LDLc in individuals of African descendent resulting from frequent nonsense mutations in PCSK9. Nat Genetics. 2005;37:161-5.

29. Lavigne PM, Karas RH. The current state of niacin in cardiovascular disease prevention: a systematic review and meta-regression. J Am Coll Cardiol. 2013;61:440-6.

30. Sabatine MS, Giugliano RP, Keech AC, et al., For the FOURIER steering committee and investigators. Evolocumab and clinical outcomes in patients with cardiovascular disease. New Engl J Med. 2017. Published on line, march 17.

31. Schwartz GG, Bessac L, Berdan LG, et al. Effect of alirocumab, a monoclonal antibody to PCSK9, on long-term cardiovascular outcomes following acute coronary síndromes: rationales and design of the ODYSSEY OUTCOMES trial. Am Heart J. 2014;168, 682-9.e1.

32. Karen MJ, Sabatine MS, Giugliano RP, et al. Long-term lowdensity lipoprotein cholesterol-lowering efficay, persistence, and safety of evolocumab in treatment of hypercholesterolemia. Results up to 4 years from the open-label OSLER 1 extension study. JAMA Cardiol. 2017. Published on line, March 14.

33. Giugliano RP, Mach F, Zavitz K, et al. EBBINGHAUSS: a cognitive study of patients enrolled in the FOURIER trial. ACC-66th anual scientific sesión-late-breaking clinical trial. 2017.

34. Robinson JG, Farnier M, Krempf M, et al. Efficacy and safety of alirocumab in reducing lipids and cardiovascular events. N Engl J Med. 2015;372:99-1489.

35. Robinson JG, Rosenson RS, Farnier M, et al. Safety of very low density lipoprotein colesterol levels with alirocumab. Pooled data from randomized trials. J Am Coll Cardiol. 2017;69:471-82.

36. Nissen SE, Tuzcu PM, Schoenhagen P, et al. Effect of intensive compared with moderate lipid-lowering therapy on progression of coronary atherosclerosis. The REVERSAL Trial. JAMA. 2004;29:80-1071.

37. Nissen SE, Nicholls SJ, Sipai I, et al. Effect of very high-intensity statin therapy on regression of coronary aterosclerosis. The ASTEROID Trial. JAMA. 2006;29:65-1556.

38. Stephen J, Nicholls M, Ballantyne ChM, et al. Effect of two intensive statins regimens on progression of coronary disease. New Eng J Med. 2011;365:87-2078.

39. Tsujita K, Sugiyama S, Sumida H, et al. Impact of dual lipidlowering strategy with ezetimibe and atorvastatin on coronary plaque regression in patients with percutaneous coronary intervention. The multicenter randomized controlled PRECISE-IVUS Trial. JACC. 2015;66:495-507.

40. Nicholls SJ, Puri R, Anderson T, et al. Effect of evolocumab on progression of coronary disease in statin-treated patients. The GLAGOV randomized clinical trial. JAMA. 2016;316: 84-2373.

41. Puri R, Nissen S, Shao M, et al. Coronary ateroma volumen and cardiovascular events during maximally intensive statin therapy. Eur Heart J. 2013;34:90-3182.

42. Landmesser U, Chapman MJ, Farnier M, et al. European Society of Cardiology/European Atherosclerosis Society task force consensus statement on proprotein convertase subtilisin/Kesin type 9 inhibitors: practical guidance for use in patients at very high cardiovascular risk. Eur Heart J. 2016;17. Published on line, october.

43. Alkindi M, Siminovitch KA, Gupta M, et al. Monoclonal antibodies for the treatment of hypercholesterolemia: targeting PCSK9. Canadian J Cardiol. 2016;32:60-1552.

44. Orringer CE, Jacobson JA, Saseen JJ, et al. Update on the use of PCSK9 inhibitors in adults: recomendations from an expert panel of the National Lipid Association. J Clinical Lipidol. 2017. Published on line, may 2.

45. Merchan A, Ruiz A, Campo R, et al. Hipercolesterolemia familiar: artículo de revisión. Rev Colomb Cardiol. 2016;23(S4):4-26.

46. Ference B, Majeed F, Penumetcha R, et al. Effect of naturally random allocation to lower low-density lipoprotein cholesterol on the risk of coronary heart disease mediated by polymorphism in NPC1L1. HMGCR or both. A $2 \times 2$ factorial mendelian randomization study. JACC. 2015;65:61-1522.

47. Ference BA, Ginsberg HN, Graham I, et al. Low-density lipoprotein cause atherosclerotic cardiovascular disease. 1. Evidence from genetic, epidemiologic, and clinical studies. A consensus from the European Atherosclerosis Society consensus panel. Eur Heart J. 2017. Published on line, march 8.

48. Perez de Isla L, Alonso R, Watts GF, et al. Attainment of LDL-cholesterol treatment goals in patients with familial hypercholesterolemia. 5-year SAFEHEART registry follow-up. J AM Coll Cardiol. 2016;67:85-1278. 